\title{
Tardígrados asociados a una plantación de cítricos de traspatio en la comunidad de El Palmar en Quintana Roo, México
}

\author{
Tardigrades associated with a backyard citrus plantation \\ in El Palmar community in Quintana Roo, Mexico
}

\begin{abstract}
Abril Anguas-Escalante*凶, Wilbert Andrés Pérez-Pech**, Roberło Guidetti***, Leopoldo Querubín Cutz-Pool**, Héctor Ortiz-León***

Anguas-Escalante, A., Pérez-Pech, W. A., Guidetti, R., Cutz-Pool, L. Q., \& Ortiz-León, H. (2018). Tardígrados asociados a una plantación de cítricos de traspatio en la comunidad de El Palmar en Quintana Roo, México. Investigación y Ciencia de la Universidad Autónoma de Aguascalientes, 26(73), 20-26.
\end{abstract}

El presente estudio provee el registro de tardígrados de líquenes en una plantación de cítricos de traspatio de la comunidad de El Palmar en el estado de Quintana Roo, México. Se proporciona la diagnosis de los géneros Ramazzottius, Mesobiotus y Milnesium. Se discute sobre la riqueza y abundancias de tardígrados en el estado. Se proporciona un cuadro comparativo de los caracteres más relevantes de los tres géneros.

\section{ABSTRACT}

This study provides records of tardigrades in lichen in a backyard plantation of citrics of a rural community in Quintana Roo, Mexico. A diagnosis is given of Ramazzottius, Mesobiotus and Milnesium genera are given. The richness of species and abundance of tardigrades in Quintana Roo are discussed. A comparative table of the most relevant characteristics of the three genera is proposed.

Palabras clave: Tardigrada; liquen; agroecosistema. Keywords: Tardigrada; lichen; agroecosystem.

\section{Recibido: 21 de marzo de 2017, aceptado: 30 de octubre de 2017}

* El Colegio de la Frontera Sur. Laboratorio de Pesquerías B13, Ave. Centenario km 5.5, C. P. 77014, Chetumal, Quintana Roo, México. Correo electrónico: abrilanguas@hotmail.com

** Departamento de Ingeniería Química y Bioquímica, Instituto Tecnológico de Chetumal, Tecnológico Nacional de México. Av. Insurgentes No. 330, C. P. 77013, Chetumal, Quintana Roo, México. Correo electrónico: pilon_45@hotmail.com; cutzpool@yahoo.com; oilh@yahoo.com.mx

*** Department of Life Sciences, University of Modena and Reggio Emilia. Via Campi 213/D, 41125, Modena, Italy. Correo electrónico: roberto. guidetti@unimore.it

$凶$ Autor para correspondencia
Los tardígrados son micrometazoarios hidrófilos con simetría bilateral, generalmente presentan tallas de 50 a $1500 \mu \mathrm{m}$, sin embargo, se ha documentado que Milnesium eurystomum Maucci, 1988, puede llegar a medir más de los $2 \mathrm{~mm}$ de longitud (Guil, 2008; Kaczmarek, Bartels, Roszkowska, \& Nelson, 2015; Marley, Mclnnes, \& Sands, 2011). El cuerpo de estos animales se divide en cinco pseudosegmentos, el primero corresponde a la cabeza. Los cuatro restantes conforman el cuerpo, donde cada cual contiene un par de patas (Marley et al., 2011; Ramazzotti \& Maucci, 1983). En la mayoría de los eutardígrados, así como algunos heterotardígrados (limnoterrestes) pueden existir garras insertadas directamente a cada pata (Pilato \& Binda, 2010); mientras que en los Arthrotardigrada (tardígrados marinos) existen individuos que presentan dígitos en sus patas, sobre los cuales se originan garras o discos adhesivos (Fontoura, Bartels, Jørgensen, Kristensen, \& Hansen, 2017).

Los tardígrados son considerados verdaderos cosmopolitas, pues se encuentran distribuidos prácticamente en todo el planeta, en ambientes terrestres y acuáticos (Glime, 2013). En su entorno son depredadores de nemátodos (SánchezMoreno, Ferris, \& Guil, 2008) y también pueden ser depredados por colémbolos (Palacios-Vargas \& Vidal-Acosta, 1994). En los últimos 25 años se ha observado un incremento en la descripción de nuevas especies de tardígrados (Degma, Bertolani, \& Guidetti, 2014). Sin embargo, su conocimiento sigue considerándose incipiente (Guil \& Cabrero- 


\section{InVESTIGACIÓn Y CIERCIA DE LA UחIVERSIDAD AUTÓnOMH DE RGUASCALIEחTES}

Sañudo, 2007). Lo anterior pudo ser constatado por Bartels, Apodaca, Mora, y Nelson (2016) al estimar la diversidad global de tardígrados. En dicho trabajo se determinó que el phylum Tardigrada además de ser pobre en especies, también es un grupo muy poco estudiado.

En México, el estudio sobre los tardígrados se remonta desde 1911 y la mayoría de los registros, al igual que los trabajos de investigación desarrollados en el país han sido esporádicos y muchas de las veces no son publicados (Hoffman \& Jiménez, 1994; Kaczmarek, Michalczyk, \& Mclnnes, 2014). Hasta ahora se han registrado 16 géneros y 43 especies de tardígrados en 11 estados del país (Beasley, 1972; Beasley, Kaczmarek, \& Michalczyk, 2008; Claps \& Rossi, 2002; Heinis, 1911; Kaczmarek, Diduszko, \& Michaelczyk, 2011, Kaczmarek et al., 2014; May, 1948; Moreno-Talamantes, Roszkowska, Guayasamín, Flores Maldonado, \& Kaczmarek, 2015; Pilato \& Lisi, 2006; Schuster, 1971). En Quintana Roo, los esfuerzos por conocer la riqueza genérica de tardígrados son recientes. El primer estudio fue realizado en 2016, donde Pérez-Pech, Cutz-Pool, Guidetti, y BlancoPiñón (2016) proporcionan los primeros registros genéricos de estos organismos en un área urbana en la ciudad de Chetumal.

El estudio en áreas rurales, así como en sus ecosistemas modificados, tales como áreas de cultivo es importante, ya que permite conocer la riqueza faunística asociada a estos ambientes (Stupino, lermanó, Gargoloff, \& Bonicatto, 2014). Actualmente, el conocimiento de tardígrados en comunidades rurales es aún escaso. Sin embargo, los que se han realizado han demostrado que estos ambientes pueden albergar una alta diversidad de tardígrados (Johansson, Calloway, Miller, \& Linder, 2011 ; Meininger, Uetz, \& Snider, 1985; Meyer, Hinton, \& Samletzka, 2013; Séméria, 1981). El hecho de que la humedad en estos lugares no disminuye drásticamente permite el establecimiento del liquen y el musgo, microecosistemas que amortiguan la pérdida de humedad permitiendo la presencia de estos organismos (Glime, 2013). También se ha comprobado el valor como indicador de calidad ambiental de algunas especies de tardígrados al evaluar la diversidad de los mismos en ambientes urbanos y rurales en Argentina (González-Reyes et al., 2016).

El presente estudio tiene como objetivo obtener la riqueza genérica de tardígrados asociada a muestras de liquen colectadas en arboles de una plantación de cítricos de traspatio, en la comunidad rural El Palmar en el municipio de Othón Pompeyo Blanco en el estado de Quintana Roo, México (figura 1). Con ello se busca contribuir al conocimiento de tardígrados en áreas rurales, así como generar nuevos registros genéricos para el estado de Quintana Roo.

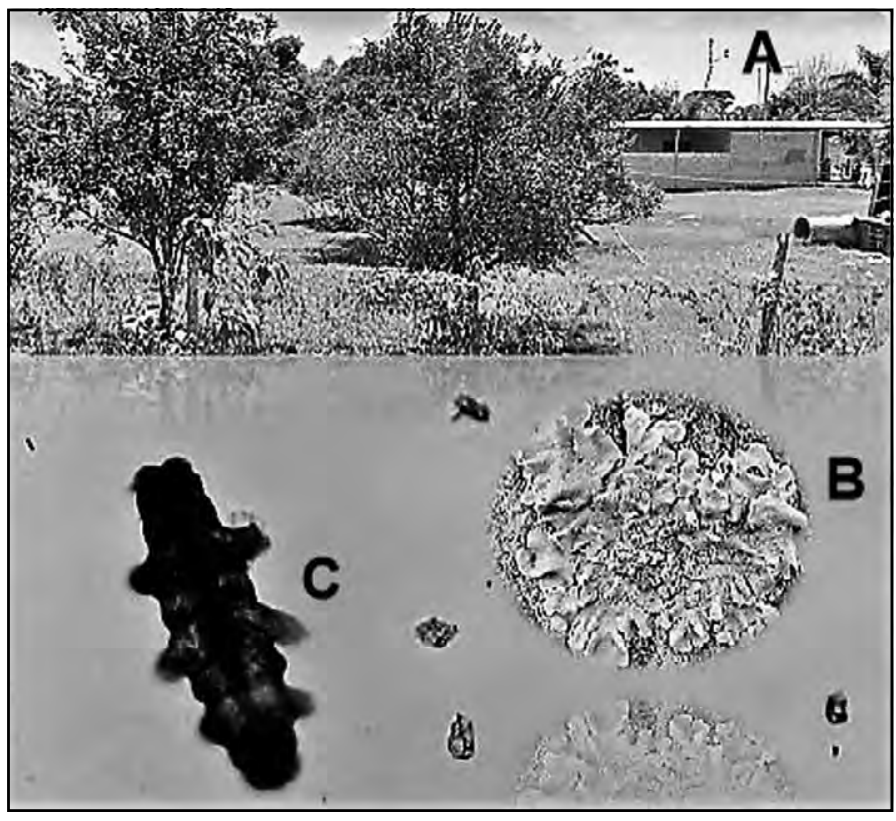

Figura 1.(A) Plantación de traspatio en la comunidad de palmar donde se realizó el estudio. (B) Ambiente muestreado. (C) Espécimen observado.

Elaboración propia.

\section{MATERIALES Y MÉTODOS}

El presente estudio se realizó en una plantación de cítricos en un traspatio ubicado en la comunidad "El Palmar" (18 $8^{\circ} 26^{\prime} 48^{\prime \prime} \mathrm{N}, 88^{\circ} 31^{\prime} 47^{\prime \prime} \mathrm{W}, 10 \mathrm{~m}$ de altitud) en el Municipio de Othón P. Blanco, Quintana Roo. La toma de muestras consistió en la colecta de liquen en 11 árboles realizado el 3 de septiembre de 2016. El liquen se obtuvo desprendiéndolo con una navaja desde la corteza del árbol. Las muestras fueron tomadas a $1 \mathrm{~m}$ de altura del suelo, se realizó el desprendimiento de $10 \mathrm{~cm}^{2}$ de liquen por árbol. El resguardo de las muestras fue en sobres de papel encerado, donde se anotaron los datos de colecta: nombre del colector, fecha, lugar y número de árbol. Las muestras fueron procesadas en el Laboratorio de Zoología en el Instituto Tecnológico de Chetumal, los organismos colectados fueron montados e identificados en el laboratorio de pesquerías en El Colegio de la Frontera Sur (ECOSUR) unidad Chetumal. Para analizar las muestras, fueron 


\section{INVESTIGAGIÓก Y CIECEIA DE LA UחIVERSIDAD AUTÓกOMA DE AGUASCALIERTES \\ DE nGUnSCRLE}

colocadas en cajas de Petri e hidratadas con agua potable durante $1 \mathrm{~h}$. Una vez sometido a una humedad adecuada, el liquen fue revisado utilizando un microscopio Stemi DV4 Carl Zeiss ${ }^{\circledR}$ de disección con el fin de separar y contabilizar a los tardígrados encontrados. Para realizar la identificación de los géneros se realizaron preparaciones permanentes empleando el líquido de Hoyer como medio de montaje. Para la identificación taxonómica se realizaron observaciones y toma de fotografías digitales a través del microscopio óptico VELAB B-1T®. Se emplearon claves de identificación especializada (Nelson, Guidetti, \& Rebecchi, 2010; Pilato \& Binda, 2010; Ramazzotti \& Maucci, 1983). De igual manera, se estructuró un cuadro comparativo con las características diferenciales de los géneros identificados. Los caracteres tomados en cuenta fueron la configuración de las garras y el aparato bucal faríngeo (tabla 2). Los organismos colectados e identificados se depositaron en el Laboratorio de Zoología en el Instituto Tecnológico de Chetumal.

\section{RESULTADOS}

Se obtuvieron un total de 717 individuos pertenecientes a tres familias y tres géneros de la clase Eutardigrada. Los 11 árboles muestreados tuvieron presencia de tardígrados, el promedio de tardígrados recolectados por muestra fue de 65 con una desviación estándar de 215. La abundancia máxima fue de 250 individuos en el árbol 10 y la mínima de 18 individuos en el árbol 5 (tabla 1). Los tres géneros registrados son nuevos para el estado. El género Ramazzottius fue el mejor representado en cuanto a su abundancia, con 592 organismos, es decir, 83\% del total de los ejemplares colectados (figura 2, tabla 1). Este género también fue registrado en todas las muestras de líquenes embebidos en los árboles de los cítricos muestreados. Se obtuvo un cuadro comparativo con los caracteres diferenciales de los géneros identificados en el presente estudio (tabla 2).

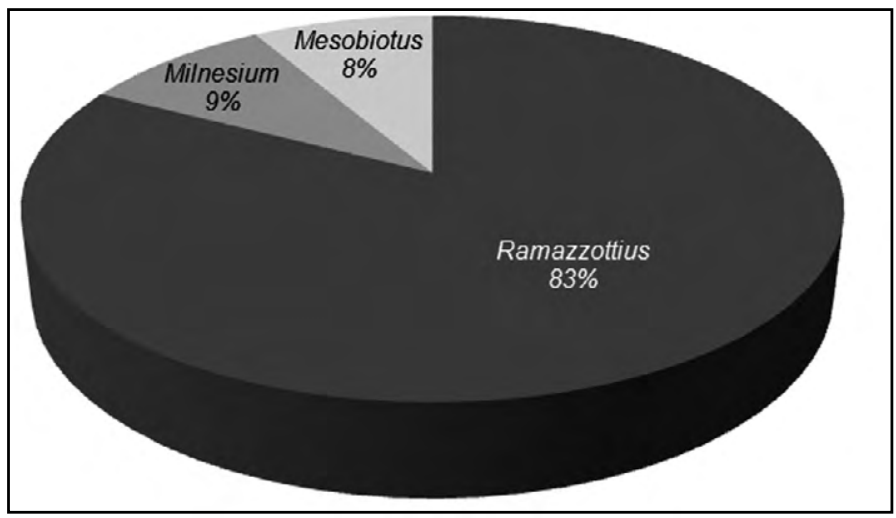

Figura 2. Distribución de la abundancia relativa de tardígrados asociados a los líquenes de cítricos de un traspatio en la comunidad de El Palmar, Quintana Roo.

Elaboración propia.

\section{Diagnosis de géneros}

Género: Milnesium (Milnesiidae) Doyère 1840 (figura

3A). Tardígrados con un par de papilas cefálicas y seis papilas peribucales. Garras del tipo Milnesiidae (la ramificación primaria y secundaria completamente separadas) (figura 3C). Aparato bucal-faríngeo del modelo Milnesium (figura $3 \mathrm{~B}$ ), con un amplio tubo bucal en las especies conocidas. Bulbo faríngeo elongado sin apófisis y placoides ausentes. Largas láminas peribucales.

Tabla 1

Clase, Familias, Géneros y abundancias de tardígrados colectados por árbol en el área de estudio

\begin{tabular}{|c|c|c|c|c|c|c|c|c|c|c|c|}
\hline Clase/Familia/Género & Al & A2 & A3 & A4 & A5 & A6 & A7 & A8 & A9 & A10 & A11 \\
\hline \multicolumn{12}{|l|}{ Eutardigrada } \\
\hline \multicolumn{12}{|l|}{ Ramazzottiidae } \\
\hline Ramazzottiius & 54 & 34 & 42 & 1 & 15 & 31 & 38 & 25 & 20 & 219 & 113 \\
\hline \multicolumn{12}{|l|}{ Milnessiidae } \\
\hline Milnesium & 7 & 4 & & & & 2 & 3 & 4 & 1 & 31 & 12 \\
\hline \multicolumn{12}{|l|}{ Macrobiotidae } \\
\hline Mesobiotus & 2 & & 18 & 35 & 3 & 1 & 1 & 1 & & & \\
\hline
\end{tabular}

Nota: $\mathrm{A} 1-11$, árboles donde los tardígrados fueron colectados.

Elaboración propia. 


\section{InVESTIGACIÓn Y CIEnCIA DE LA UחIVERSIDAD AUTÓnOMA DE RGUASCALIENTES}

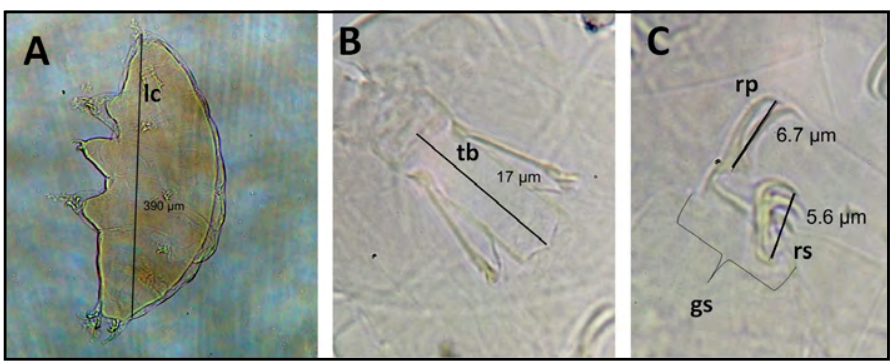

Figura 3. (A) Cuerpo de Milnesium; (B) Aparato bucal-faríngeo Milnesium; (C) Garras tipo Milnesiidae-gs garras separadas, IC: longitud corporal, tb: tubo bucal rp: ramificación primaria, rs: ramificación secundaria.

Fotografías tomadas por el equipo de estudio.

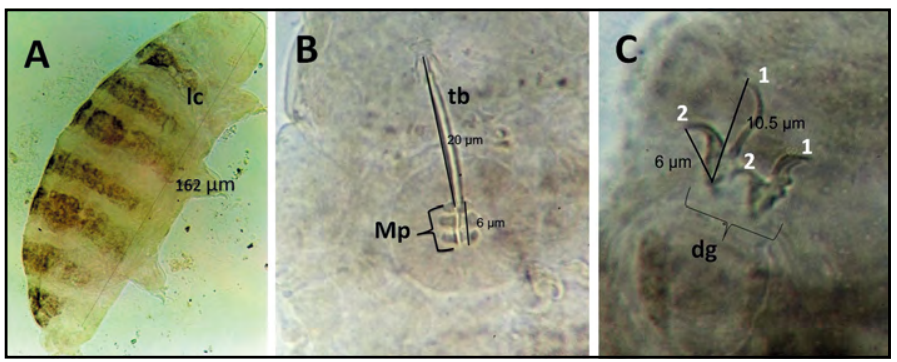

Figura 4. (A) Cuerpo de Ramazzottius; (B) aparato bucal del tipo Hypsibius, variante Ramazzottius; (C) garras del tipo Ramazzottius-2, 1, 2, 1 configuración asimétrica de las diplogarras, dg: diplogarras, tb: tubo bucal, Mp: macroplacoides, lc: longitud corporal. Fotografías tomadas por el equipo de estudio.

Género: Ramazzottius Binda y Pilato, 1986 (figura 4A). Tardígrados con un par de órganos elípticos presentes en la cabeza. Diplogarras (garras unidas) del tipo Ramazzottius con configuración asimétrica 2, 1, 2, 1 ramificaciones: secundaria, primaria, secundaria, primaria (figura 4C). Diplogarras externas con una sección basal más larga que la ramificación secundaria, ramificación primaria muy larga y delgada conectada con un tracto basal delgado y flexible. Diplogarras internas más cortas y robustas unificadas en un único elemento rígido. Aparato bucal-faríngeo de tipo Hypsibius, variante Ramazzottiius (figura 4B). Apófisis faríngea y dos placoides presentes. Estilete furcado de la forma tradicional. Lúnulas de garras reducidas y muy pequeñas presentes o ausentes. Huevos puestos libremente, se caracterizan por presentar procesos en su pared.

Género: Mesobiotus (Macrobiotidae) Vecchi, Cesari, Bertolani, Jönsson, Rebecchi \& Guidetti, 2016 (figura 5A). Macrobiotoideos con diplogarras de tipo-"Y" con arreglo simétrico (ramificaciones: secundaria, primaria, primaria, secundaria) del modelo Mesobiotus (figura $5 \mathrm{C}$ ), con un tracto común caracterizado por un septo interno que define una parte distal. Cutícula sin poros. Aparato bucal-faríngeo con un anillo bucal que contiene 10 láminas peribucales. Tubo bucal rígido, tres macroplacoides redondeados dispuestos a lo largo de una línea curva y un microplaciode. La distancia entre el microplacoide y el tercer macroplacoide es reducida (el espacio entre estas es menor que la longitud del microplacoide) (figura 5B). Huevos puestos libremente, caracterizado por procesos cónicos o hemisféricos, generalmente con puntas.

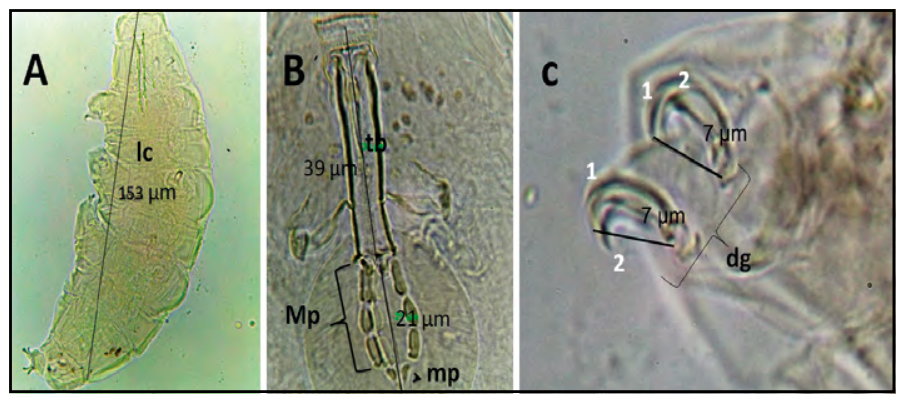

Figura 5. (A) Cuerpo de Mesobiotus; (B) aparato bucal-faríngeo de tipo Mesobiotus; (C) diplogarras-2, 1, 1, 2 configuración simétrica de las diplogarras, dg: diplogarras, tb: tubo bucal, Mp: macroplacoides, mp: microplacoides.

Fotografías tomadas por el equipo de estudio.

Tabla 2

Diferencias de caracteres de Ramazzottius, Milnesium y Mesobiotus

\begin{tabular}{|c|c|c|c|c|}
\hline Género & Garras & Placoides & $\begin{array}{c}\text { Configuración de } \\
\text { garras }\end{array}$ & $\begin{array}{l}\text { Configuración de } \\
\text { placoides }\end{array}$ \\
\hline Ramazzottius & $\begin{array}{c}\text { Unidas } \\
\text { (figura } 4 \mathrm{C} \text { ) }\end{array}$ & Presentes & $\begin{array}{l}\text { Asimétrica }(1,2,1,2) \\
\text { (figura } 4 \mathrm{C})\end{array}$ & $\begin{array}{l}2 \text { macroplacoides } \\
\text { (figura } 4 \mathrm{~B})\end{array}$ \\
\hline Milnesium & $\begin{array}{l}\text { Separadas } \\
\text { (figura 3C) }\end{array}$ & $\begin{array}{l}\text { Ausentes } \\
\text { (figura 3B) }\end{array}$ & - & - \\
\hline Mesobiotus & $\begin{array}{l}\text { Unidas } \\
\text { (figura } 5 \mathrm{C} \text { ) }\end{array}$ & Presentes & $\begin{array}{l}\text { Simétrica }(2,1,1,2) \\
\text { (figura } 5 \mathrm{C})\end{array}$ & $\begin{array}{l}3 \text { macroplacoides } \\
1 \text { microplacoide (figura 5C) }\end{array}$ \\
\hline
\end{tabular}

Nota: Elaboración propia. 
IIVESTIGACIÓn Y CIERCIA DE LA UNIVERSIDAD AUTÓNOMA DE AGUASCALIERTES
La biodiversidad constituye la base de la vida y de la sustentabilidad de los agroecosistemas, por lo cual las áreas de cultivo proveen de nichos para diferentes organismos (Stupino et al., 2014). El ser humano al emplear las áreas agrícolas para obtener recursos y cubrir sus necesidades básicas genera ambientes modificados en los cuales algunos grupos faunísticos son reemplazados y muchos otros logran adaptarse, tal es el caso de los tardígrados y una variedad de microartrópodos (Pérez-Pech \& Cutz-Pool, 2015). En Quintana Roo se han reportado ácaros, colémbolos, y una variedad de artrópodos como excelentes colonizadores de ambientes modificados (Cutz-Pool, Alcocer-May, Ortiz-León, Castro-Pérez, \& LópezChan, 2015; Cutz-Pool, Escalante-Poot, \& OrtizLeón, 2016; Pérez-Pech, Cutz-Pool, \& Martin-Cerón, 2014), entre estos las áreas agrícolas (Pérez-Pech \& Cutz-Pool, 2015). El presente estudio aporta al conocimiento de la fauna agroecosistémica de la entidad con la inclusión de los tardígrados a la lista de organismos presentes en los cultivos.

El liquen, musgo, y la hojarasca, son los ecosistemas preferidos en ambientes naturales por los géneros registrados (Kaczmarek et al., 2014). En ambientes modificados (urbanos y rurales) los tardígrados generalmente optan por habitar liquen y musgo, lo que relaciona su diversidad con la salud ambiental de estos sitios (Glime, 2013; GonzálezReyes et al., 2016); por lo que algunas especies han sido catalogadas como indicadoras de calidad ambiental (Macrobiotus hufelandi, Schultze, 1834), o detectoras de ambientes urbanos, es el caso de Milnesium (González-Reyes et al., 2016). En el presente trabajo los tardígrados se encontraron asociados a liquen en árboles de cítricos en una plantación de traspatio en la comunidad de El Palmar. Milnesium fue registrado durante el estudio. Sin embargo, sus abundancias fueron relativamente bajas respecto a Ramazzottius, un género que en estudios anteriores ha mostrado altas abundancias en este mismo ambiente, frente a otras especies de tardígrados y otros micrometazoarios (Šatkauskienè, 2012).

Los géneros reportados en el presente trabajo, junto a los documentados por Pérez-Pech etal. (2016), suman un total de seis géneros para Quintana Roo, procedentes de distintos biotopos. La particularidad de los estudios sobre tardígrados en el estado de Quintana Roo radica en que han explorado la diversidad genérica en ambientes modificados (urbanos yrurales), los cuales han sido pocoestudiados en el mundo (Meyer et al., 2013). Los trabajos realizados han demostrado que estos ambientes albergan una variedad de microecosistemas que favorecen la supervivencia de los tardígrados, por lo que pueden presentar una notable riqueza genérica y específica (Johansson et al., 2011; Meininger et al., 1985; Meyer et al., 2013). Es destacable que aun cuando el esfuerzo de muestreo entre este trabajo y el realizado por Pérez-Pech et al. (2016) no fue el mismo, la riqueza genérica documentada en el presente estudio es similar a la reportada en sitios urbanos de la ciudad de Chetumal, así como que algunos géneros estuvieron mejor representados que otros. Doryphoribius fue el mejor representado en el área urbana de Chetumal con $94 \%$ de abundancias con respecto a Macrobiotus y Echiniscus, quienes representaron $6 \%$, respectivamente. En el presente estudio, el género Ramazzottius representó $83 \%$ del total de las abundancias, mientras que Milnesium y Mesobiotus $17 \%$ (tabla 1, figura 2).

Cabe señalar que el número de muestras estudiadas por Pérez-Pech et al. $(2016)$ fueron mucho más que en el presente trabajo, por lo que es necesario dirigir estudios con diseños de muestreo estandarizados para determinar estadísticamente las diferencias de la riqueza, abundancia y diversidad entre ambientes urbanos y rurales, lo que incluye distintos biotopos potenciales capaces de ser ocupados por los tardígrados (Meyer et al., 2013).

\section{CONCLUSIONES}

Se contribuye notablemente en el conocimiento de la fauna de tardígrados en el estado de Quintana Roo, se proporcionan tres nuevos registros genéricos. Se hace patente la necesidad de mayores esfuerzos para conocer las diferencias en diversidad de tardígrados entre áreas urbanas y rurales para ser considerados como buenos indicadores en la salud ambiental. 


\section{IIVESTIGAGIÓn Y CUERCIA DE LA UNIVERSIDAD AUTÓnOMF DE RGUASCALIERTES}

\section{Agradecimientos}

Los autores desean agradecer a la línea de investigación Biodiversidad en ecosistemas terrestres (Ecología de Artrópodos) del Instituto Tecnológico de Chetumal, a la estudiante de biología Juana Itzel Crisanto Rodríguez por el apoyo brindado para realizar la colecta de campo, al programa Preparación al Posgrado y Asistentes de Investigador
2016 del Colegio de la Frontera Sur, del cual fue becario el primer autor durante la realización del proyecto, al Dr. Alberto de Jesús Navarrete por las facilidades otorgadas para utilizar las instalaciones del Laboratorio de Pesquerías para la separación, montaje e identificación de los tardígrados, así como a los revisores del presente trabajo por sus acertadas observaciones.

\section{REFERENCIAS}

- Bartels, P. J., Apodaca, J. J., Mora, C., \& Nelson, D. R. (2016). A global biodiversity estimate of a poorly known taxon: phylum Tardigrada. Zoological Journal of the Linnean Society, 178(4), 730-736.

- Beasley, C. W. (1972). Some tardigrades from Mexico. Southwestern Naturalist, 17, 21-29.

- _Kaczmarek, L., \& Michalczyk, L. (2008). Doryphoribius mexicanus, a new species of Tardigrada (Eutardigrada, Hypsibiidae) from Mexico (North America). Proceedings of the Biological Society of Washington, 121 (1), 34-40.

- Claps, M. C., \& Rossi, G. (2002). Tardigrada. En J. LlorenteBousquets, \& J. E. Bousquets (Eds.), Biodiversidad, taxonomía y biogeografía de artrópodos de México: Hacia una síntesis de su conocimiento. Vol. III (pp. 171-186). México: Universidad Nacional Autónoma de México.

- Cutz-Pool, L. Q., Alcocer-May I. S., Ortiz-León H. J., Castro Pérez J. M. y López-Chan J. A. (2015). Riqueza genérica y preferencia de hábitat de Collembola (Hexapoda: Collembola) en el municipio de Othon Pompeyo Blanco Quintana Roo. Entomología Mexicana, 2, 161-166.

- Cutz-Pool, L. Q., Escalante-Poot, G. A., \& Ortiz-León, H. J. (2016). Arañas (Arachnida: Araneae) asociadas a dos ecosistemas contrastante en el ejido de Petcacab, Quintana Roo. Entomología mexicana, 3, 58-63.

- Degma, P., Bertolani, R., \& Guidetti, R. (2014). Actual checklist of Tardigrada species. Ver. 31. Recuperado de http://www. tardigrada. modena. unimo. it/miscellanea/Actual checklist of Tardigrada. pdf on

- Fontoura, P., Bartels, P. J., Jørgensen, A., Kristensen, R. M., \& Hansen, J. G. (2017). A dichotomous key to the genera of the Marine Heterotardigrades (Tardigrada). Zootaxa, 4294(1), 1-45.

- Glime, J. M. (2013). Tardigrades. En Bryophyte Ecology: Vol. 2. Bryological Interaction. Digital Commons@ Michigan Tech. Recuperado de www.bryoecol.mtu.edu

- González-Reyes, A., Acosta, X., Corronca, J., Rocha, M., Doma, I., \& Repp, E. Y. (2016). Evaluación de la diversidad en comunidades de tardígrados (Ecdysozoa: Tardigrada) en hábitats urbano y rural de la ciudad de Salta (Argentina). Iheringia. Série Zoologia, 106. doi: 10.1590/1678-4766e2016026

- Guil, N. (2008). New records and within-species variability of Iberian tardigrades (Tardigrada), with comments on the species from the Echiniscus blumi-canadensis series. Zootaxa, 1757(1), 1-30.

- Guil, N., \& Cabredo-Saduño, F. J. (2007). Analysis of the species description process for a littleknown invertebrate group: the limnoterrestrial tardigrades (Bilateria, Tardigrada). Biodiversity and Conservation, 16(4), 1063-1086.

- Heinis, F. (1911). Beitrag zur Kenntnis der zentralamerikanischen moss fauna. Revue Suisse de Zoologie, 19, 253-266.

- Hoffman, A., \& Jiménez, M. L. (1994). Tardígrados en México. Anales del Instituto de Biología Serie Zoología, 65(2), 297-301.

- Johansson, C., Calloway, S., Miller, W. R., \& Linder, E. T. (2011). Are urban and rural tardigrade (Tardigrada) communities distinct and determined by $\mathrm{pH}$ : A case study from Fresno County, California. The Pan-Pacific Entomologist, 87(2), 86-97.

- Kaczmarek, Ł., Bartels, P. J., Roszkowska, M., \& Nelson, D. R. (2015). The zoogeography of marine Tardigrada. Zootaxa, 4037, 1-189.

- Kaczmarek, L., Diduszko, D., \& Michalczyk, L. (2011). New records of Mexican Tardigrada. Revista Mexicana de Biodiversidad, 82, 1324-1327.

- Kaczmarek, L., Michalczyk, L., \& Mclnnes, S. J. (2014). Annotated zoogeography of non-marine Tardigrada. Part I: Central America. Zootaxa, 3763(1), 001-062.

- Marley, N. J., Mclnnes, S. J., \& Sands, C. J. (2011). Phylum Tardigrada: a re-evaluation of the Parachela. Zootaxa, 2819 , 51-64.

- May, R. M. (1948). Nouveau genre et espéces de tardigrade du Mexique: Haplomacrobiotus hermosillensis. Bullitin de la Societe Zoologique de France, 73, 95-97. 
- Meininger, C. A., Uetz, G. W., \& Snider, J. A. (1985). Variation in epiphytic microcommunities (tardigrade-lichen-bryophyte assemblages) of the Cincinnati, Ohio area. Urban Ecology, $9(1), 45-61$.

- Meyer, H. A., Hinton, J. G., \& Samletzka, C. A. (2013). Water bears in the Anthropocene: a comparison of urban and Woodland tardigrade (Phylum Tardigrada) communities in Southwestern Louisiana, USA. Journal of Limnology, 72, 123127.

- Moreno-Talamantes, A., Roszkowska, A. M., Guayasamín, P. R., Flores Maldonado, J. J., \& Kaczmarek, Ł. (2015). First record of Dactylobiotus parthenogeneticus Bertolani, 1982 (Eutardigrada: Murrayidae) in Mexico. Check List, 11 (4), 1-5.

- Nelson, D. R., Guidetti, R., \& Rebecchi, L. (2010). Tardigrada. En J. H. Thorp, \& P. A. Covich (Eds.), Ecology and Classification of North American Freshwater Invertebrates (pp 455-484). Elsevier.

- Palacios-Vargas, J. G., \& Vidal Acosta, M. V. (1994). Nuevas especies de Friesea (Collembola: Neanuridae) de reservas biológicas de México. Southwestern Entomologist, 19(3), 291291.

- Pérez-Pech, W. A., \& Cutz-Pool, L. Q. (2015). Estructura y sucesión de la fauna de artrópodos edáficos de maíz, en José María Morelos, Quintana Roo, México. Ingeniantes, 2(1), 25-28.

- Pérez-Pech, W. A., Cutz-Pool, L. Q., \& Martin-Cerón, A. (2014). Colémbolos (Hexapoda: Collembola) de una vegetación secundaria en el municipio de José María Morelos Quintana Roo, México. Entomología Mexicana, 1, 509-513.

- Pérez-Pech, W. A., Cutz-Pool, L. Q., Guidetti, R., \& Blanco-Piñón, A. (2016). Primer registro genérico de tardígrados, habitantes del área urbana de Chetumal Quintana Roo, México. Entomología Mexicana, 3, 912-918.

- Pilato, G., \& Binda, M. G. (2010). Definition of families, subfamilies, genera and subgenera of the Eutardigrada, and keys to their identification. Zootaxa, 2404, 1-54.

- Pilato, G., \& Lisi, O. (2006). Notes on some tardigrades from southern Mexico with description of three new species. Zootaxa, 1236, 53-68.

- Ramazzotti, G., \& Maucci, W. (1983). II philum Tardigrada. III edizione riveduta e aggiornata. Memorie dell'Istituto Italiano di Idrobiologia, 41, 1-1012.

- Sánchez-Moreno, S., Ferris, H., \& Guil, N. (2008). Role of tardigrades in the suppressive service of a soil food web. Agriculture, Ecosystems \& Environment, 124(3), 187-192.

- Šatkauskienè, I. (2012). Microfauna of lichen (Xanthoria parietina) in Lithuania: Diversity patterns in polluted and nonpolluted sites. Baltic Forestry, 18(2), 255-262.

- Schuster, R. O. (1971). Tardigrada from the Barranca del Cobre, Sinaloa and Chihuahua, Mexico. Proccedings of the Biological Society of Washington, $84(26), 213-224$.

- Séméria, Y. (1981). Recherches sur la faune urbaine et suburbaine des Tardigrades muscicoles et lichenicoles I. Nice-ville. Bulletin mensuel de la Société linnéenne de Lyon, 50(7), 231237.

- Stupino, S. A., lermanó, M. J., Gargoloff, N. A., \& Bonicatto, M. M. (2014). La biodiversidad en los agroecosistemas. En S. J. Sarandón, \& C. C. Flores (Eds.), Agroecología: Bases teóricas para el diseño y manejo de Agroecosistemas sustentables (pp. 131-158). La Plata: Universidad Nacional de La Plata. 